\title{
High-performance scalable Information Service for the ATLAS experiment
}

Serguei Kolos, University of California Irvine, USA

Georgios Boutsioukis, Aristotle University of Thessaloniki, Greece

Reiner Hauser, Michigan State University, USA

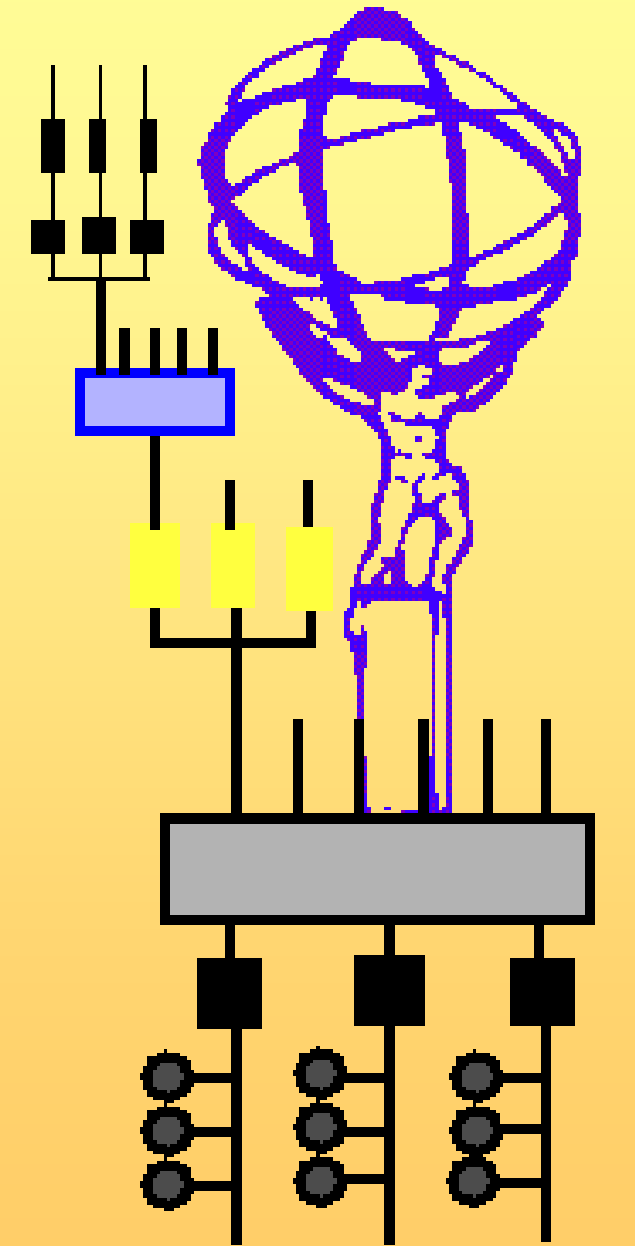

1. The ATLAS Online Monitoring System

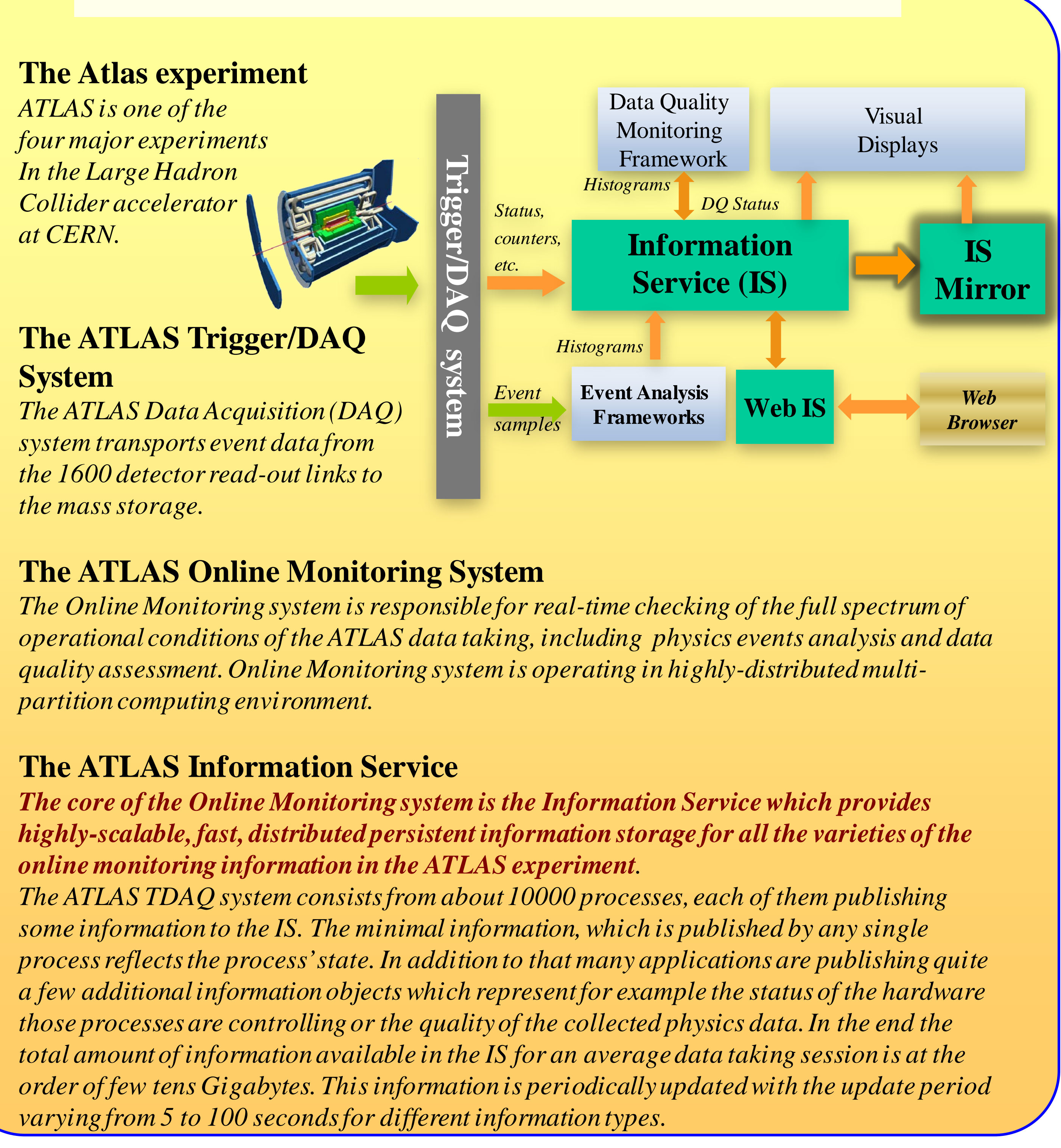

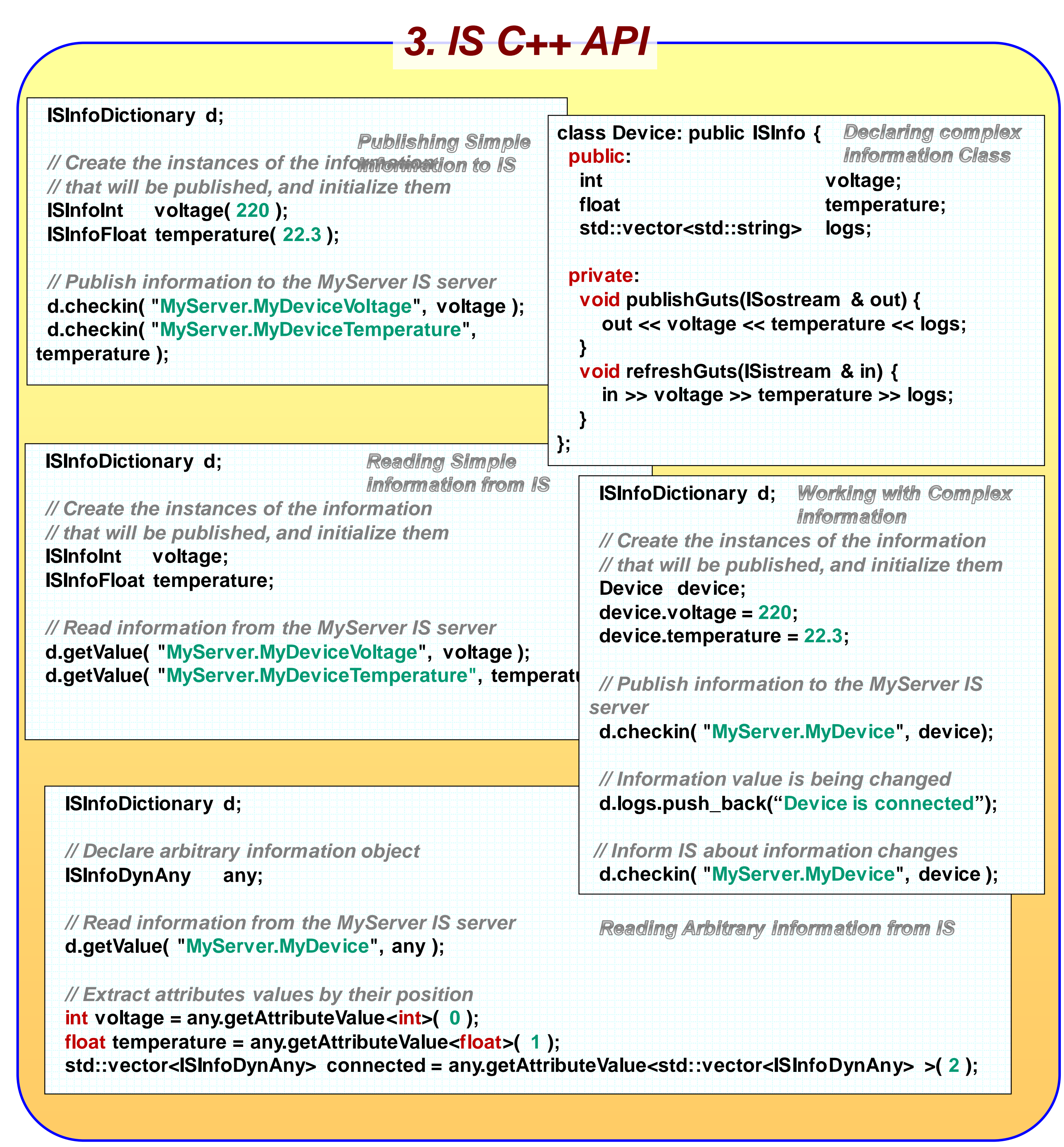

5. IS Streaming and Remote Monitoring

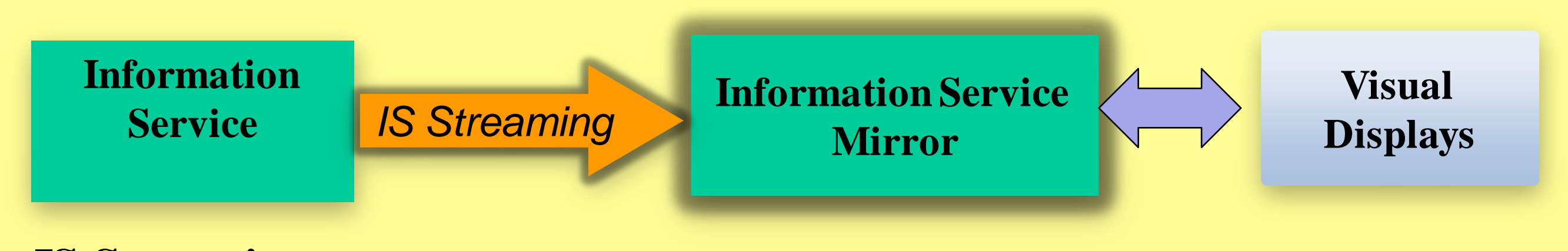

IS Streaming

IS provides the Streaming functionality. This feature streams all changes in the information held by the main IS to the secondary service, which is running in the CERN Global Public Network. The streaming works in real-time mode, thus assuring that the information
content is properly synchronized at any given moment. The average time for mirroring content is properly synchronized at any given moment,
information item is at the order of a few microseconds.

Remote Users

Remote Users
Any ATLAS member who has an active Remote Shifter role, can open $X(N X)$ sessionon one of the "ATLAS Remote Monitoring" nodes and run the same GUI displays as in the ATLAS Control Room. Those GUIs will be taking information from the IS Mirror with no impacton the data taking activity per se.

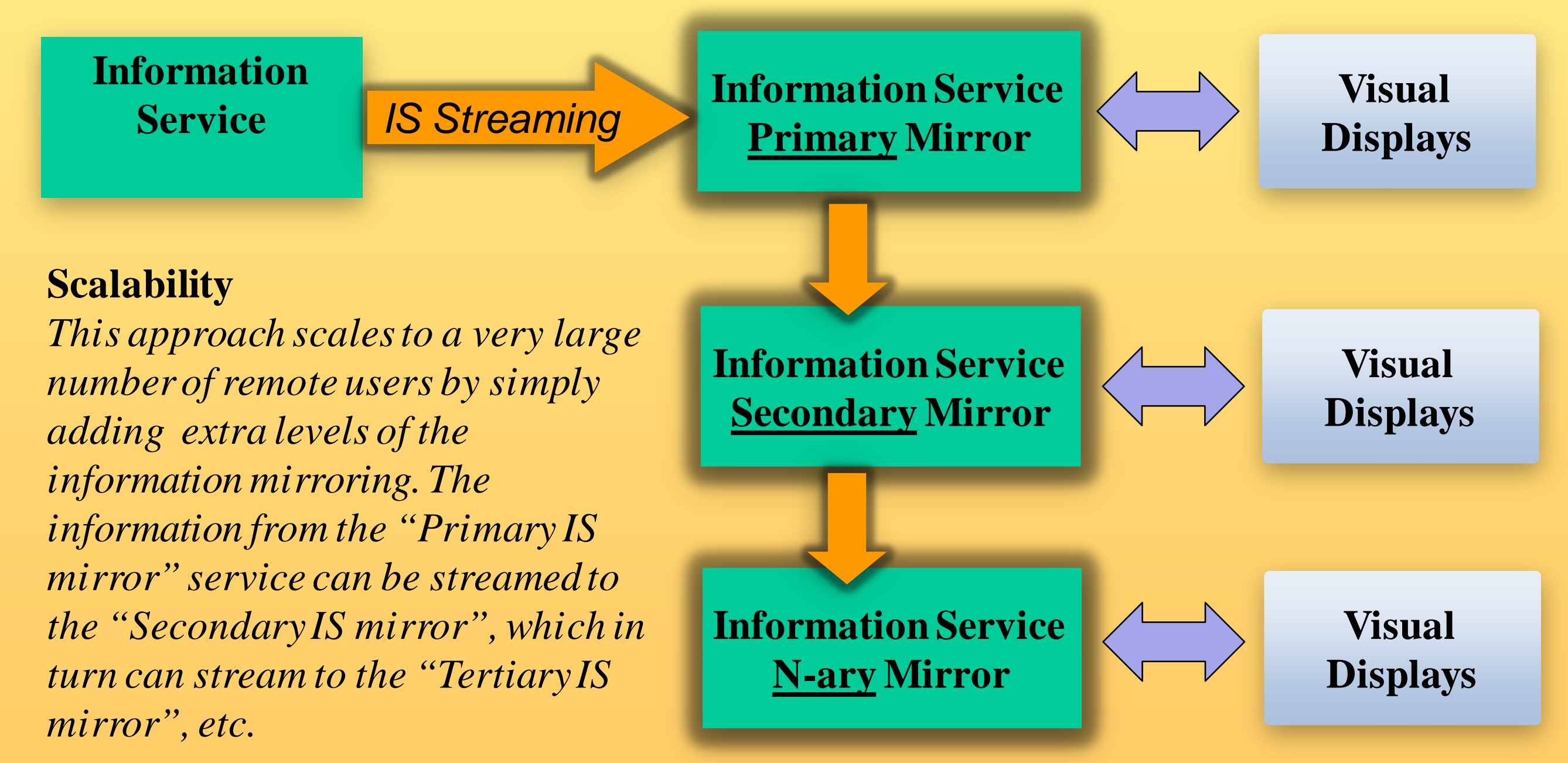

2. The Information Service

The Information Service Design

The Information Service is using the client-server architecture with the Information

applications Currently the Information Repository is implemented by a number of

processes, called IS Servers, which are distributed over several computing nodes in a location transparent way. Each server has a unique identifier, which a client application is The IS Communication Mode

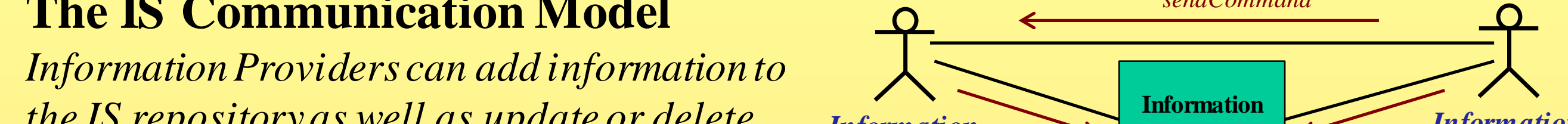

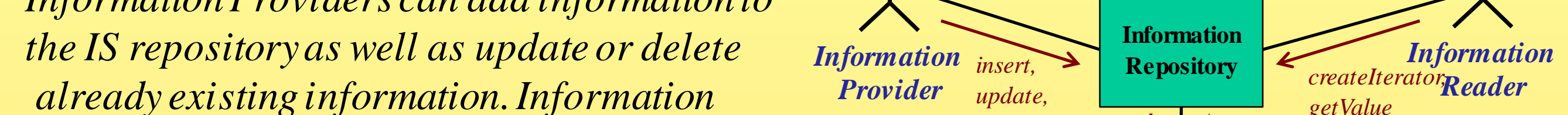
already existing information. Information Readerscan retrieve the value of an information can subscribe for the repository to be notified about changesin it Each time Provider creates, updotes or deles an information the Subscriber will be informed.

The Object Identification

Each information object has a unique name in the IS repository In the current implementation, the name is a character string which must have the following format:

The IS Meta-Information

The Information Service implementation provides access to the description of the information object classes at run-time as well as allows manipulating information whose type is not known at compilation.

The Inter-process Communication Technology The actual IS implementation is based on the Common Object Request Broker Architecture (CORBA) standard. The $\mathrm{C}++$ implementation is done on top of the omniORB CORBA broker and Java implementation is using the JacORB one. However both $\mathrm{C+}+$ and Java IS APIs are fully independent of the un The IS API

The IS Application Program Interface (API) is available in C++, Java and Python. 4. WWW interface for IS

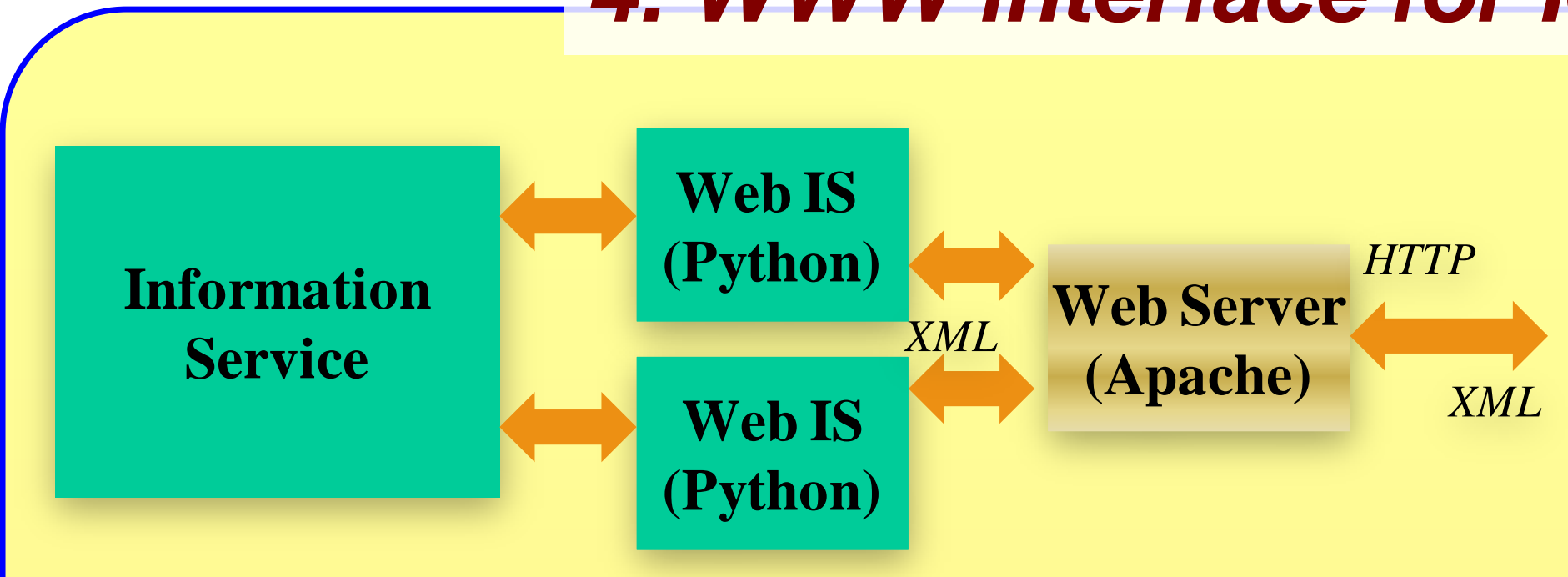

Web IS provides generic WWW interface to the Information Service. Each object in IS has a unique URL associated with it,

https://atlasop.cer ams.RunParams IS object in XML forma

This is a generic facility which is used to construct complex WEB based GUIs using CSS,

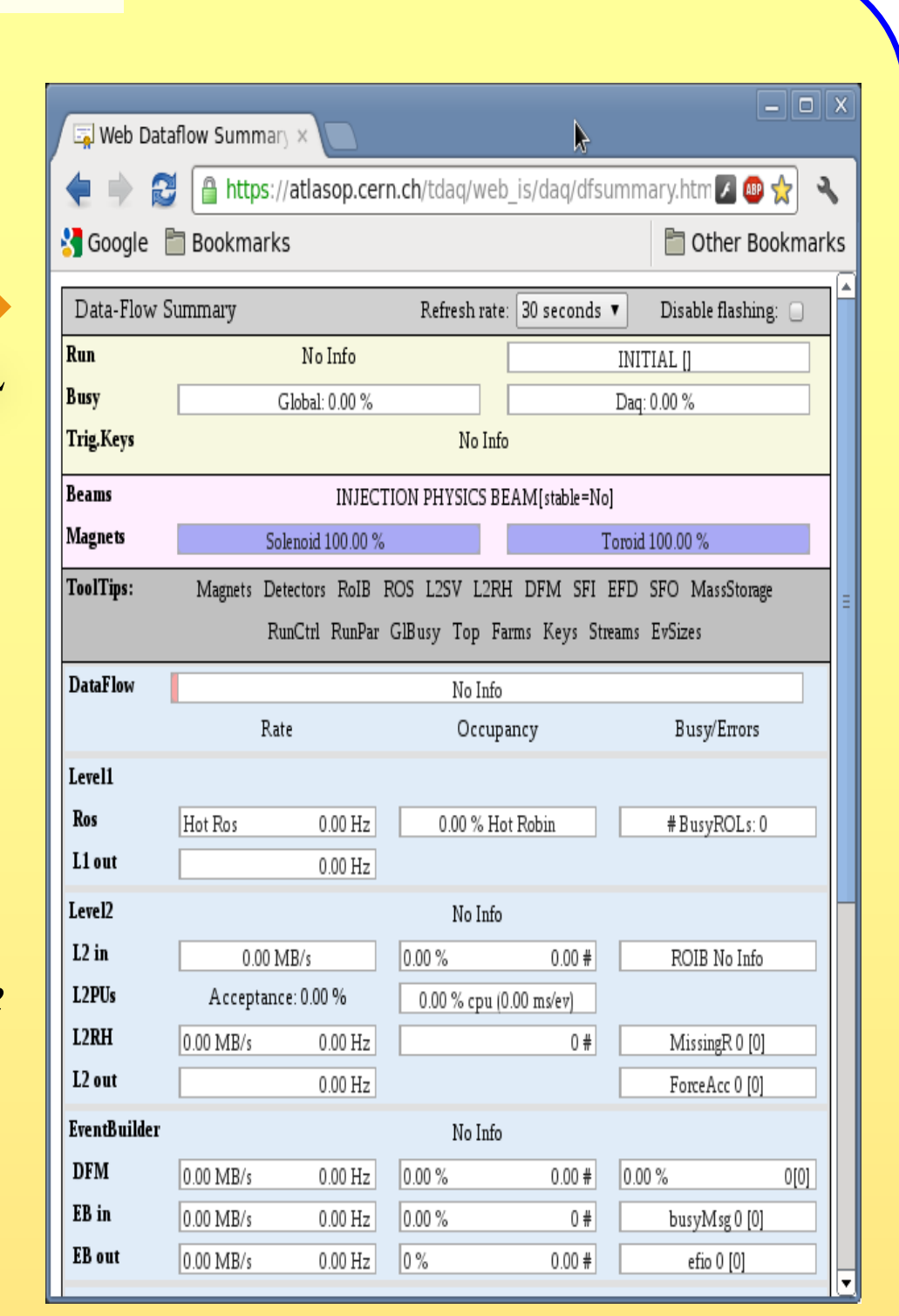

For histograms the Web IS interface can do the rasterization of images using the ROOT libraries and return those in a number of standard formats ( $P N G$, JPEG, GIF, SVG, etc.). Therefore there is no need for any HEP specific software on the client machine. This designallows users to present the info
are interested in their referable are interest
form.

The latency to access an item is determined by the HTTP access, typically in the order of $100 \mathrm{~ms}$ if one is outside of CERN. There are limits on the maximum number of concurrent requests, enforced by the servers to control the load on the online
system, which might impact data taking.

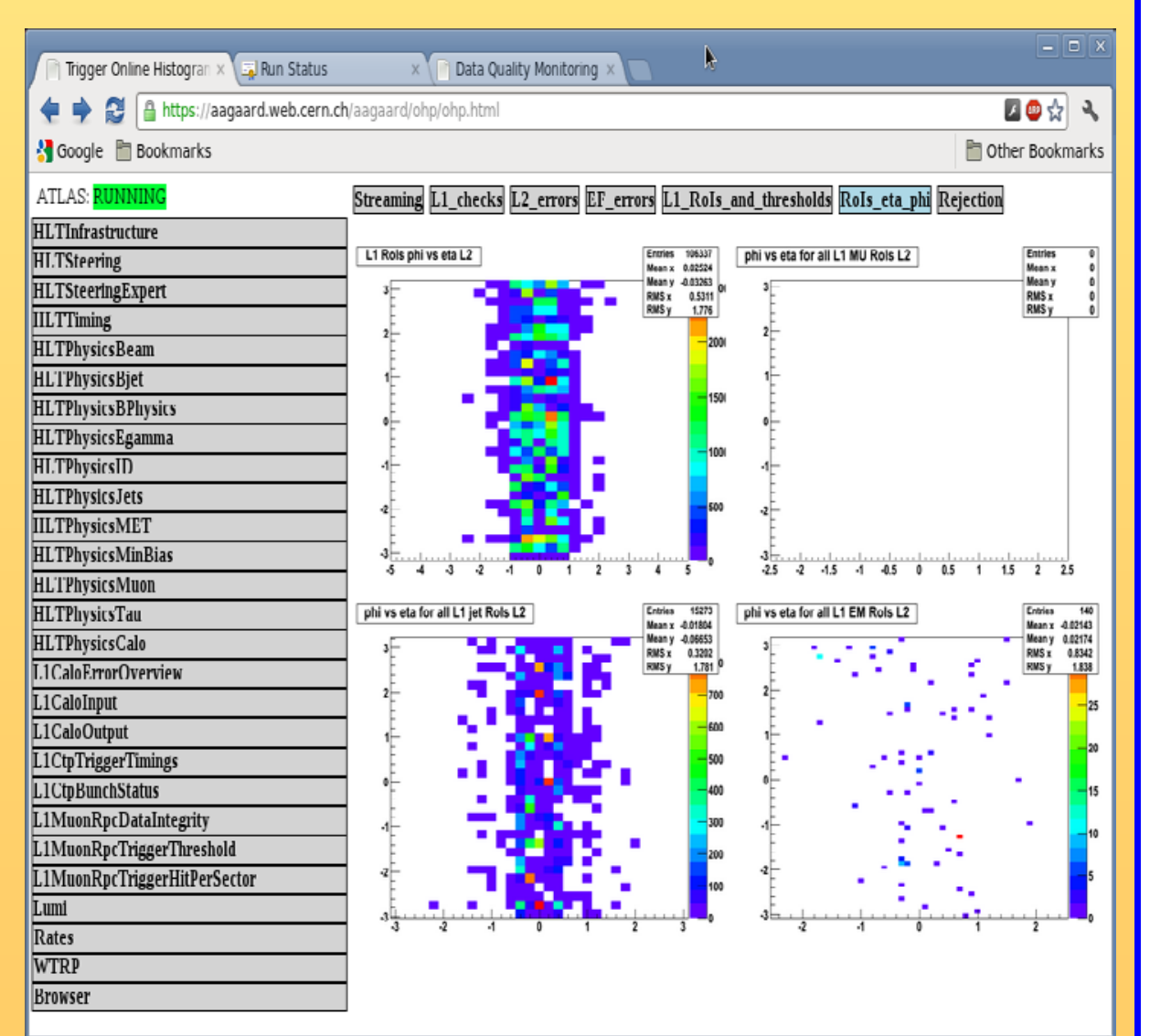

\section{IS Performance measurements}

A performance analysis of IS was conducted on the same standard hardware that was used by the ATLAS online infrastructure for the physics data taking. The IS server was running on a host with two 4-core Intel Xeon E5420 CPUs.
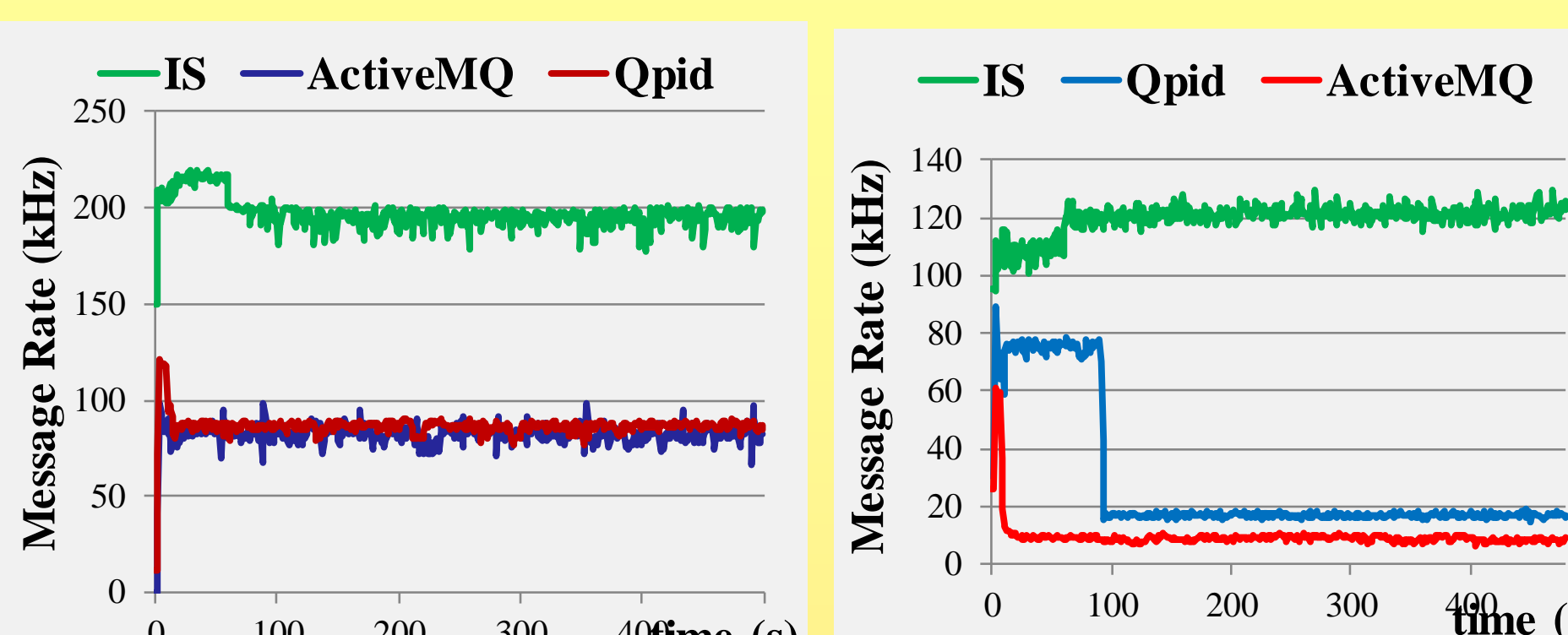

The latency, i.e. the time of passing message from the souce to all receivers, was configuration of 100 receivers and a single sender (100Hz was used

$\begin{array}{rrrr}0 & 100 & 200 & 300 \\ \text { Message delivery rate was }\end{array}$ measured for IS, Opid and ActiveMQ. The delivery res of a slow receiver. In this the sum of messages received by subscribers is accepting $\begin{array}{ll} & \text { messages at a much lower rate } \\ \text { than the rest. This forces the }\end{array}$ All systems have been tested in than the rest. This forces the saturation mode, i.e. when messaging sever to retain the maximum delivery rates was undelivered messages until it reached. For the different

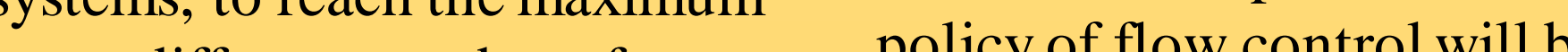
senders and receivers has been
used:

\begin{tabular}{|l|l|l|}
\hline System & Senders & Receivers \\
\hline IS & 12 & 44 \\
\hline
\end{tabular}

All three systems, IS, Qpid indemention of asis polic they are dopping holicy messages which do nose the fixed size queue.
密<smiles></smiles>

$5000 \underset{10000}{50}$

The Latency vs. Number of Receivers was measured for 9Bytes.

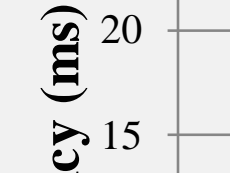

